\title{
HSCT FOR MONOCLONAL GAMMOPATHIES: MULTIPLE MYELOMA AND AMYLOIDOSIS
}

\author{
Angelo Maiolino ${ }^{1,2,3,}$ Abrahão E. Hallack Neto ${ }^{1,4,}$ Roberto Magalhães ${ }^{1,2}$, Edvan Crusoé1,5, Renata Ferreira \\ Marques Nunes ${ }^{1,6,7}$, Roberia Mendonça ${ }^{2}$, Maura R V Ikoma-Colturato ${ }^{10,11}$ Vânia T. M. Hungria ${ }^{1,8,9}$ \\ 1 Grupo Brasileiro de mieloma múltiplo (GBRAM) - 2 Faculdade de Medicina, Universidade Federal do Rio de Janeiro \\ 3 Americas Centro de Oncologia Integrado - 4 Hospital Universitário/Universidade Federal de Juiz de Fora \\ 5 Universidade Federal da Bahia - 6 Hospital Brigadeiro/Hospital de transplantes Euryclides de Jesus Zerbini \\ 7 Hospital Beneficiência Portuguesa de São Paulo - 8 Faculdade de Medicina, Santa Casa de São Paulo; \\ 9 Clinica Medica São Germano - 10 Hospital Amaral Carvalho Jau - 11 Sabin Medicina Diagnóstica
}

\section{MULTIPLE MYELOMA}

\section{INTRODUCTION}

Multiple myeloma (MM) is part of a spectrum of pathological conditions known as monoclonal gammopathies. In recent years, significant progress has been made in treating this disease, with the approval of new agents and new combinations for relapsed and newly diagnosed patients. The options must be individualized according to the patient's condition. Autologous Stem Cell Transplantation (ASCT) upfront is the standard of care for patients with good clinical conditions, and usually under the age of 75 . In Brazil and three other Latin American countries, the median age for patients eligible for ASCT was 54.7 years, and the procedure is being effectively performed in $58.6 \%$ of the patients for whom they were planned at start treatment. [1]

\section{INITIAL TREATMENT OF PATIENTS ELIGIBLE FOR ASCT}

The combination of bortezomib and dexamethasone and a third drug such as cyclophosphamide, thalidomide, or lenalidomide is the primary basis in pre-ASCT induction therapy. The pre - ASCT induction is performed for a period of 4 to 6 cycles. The pre-ASCT combinations based on bortezomib comparing with schemes without bortezomib were evaluated in a meta-analysis. Post-transplant complete remission (CR) rate, time to progression (TTP), and progression-free survival (PFS) were higher in bortezomib-based induction, with a tendency to improve overall survival (OS). [2]

The combination of bortezomib, thalidomide, and dexamethasone (VTD) was compared to bortezo- mib, cyclophosphamide, and dexamethasone (VCD) in a randomized study from the Intergroupe Francophone du Myélome (IFM). After four cycles, in an intention-to-treat analysis, $66.3 \%$ of patients in the VTD arm achieved at least a very good partial response (VGPR) vs. $56.2 \%$ in the VCD arm $(P=0.05)$. The overall response rate was significantly higher in the VTD arm than in the VCD arm (92.3\% vs. $83.4 \%$, $P=0.01)$. [3]

Thalidomide was replaced by lenalidomide in the bortezomib, lenalidomide and dexamethasone (VRD) regimen, with higher response rates and less neuropathy.

The Spanish Group PETHEMA showed excellent results with VRD in a different dose and number of cycles than those applied by the French group. VRD was used in 458 patients at induction (6 cycles) and post-ASCT consolidation ( 2 cycles). The responses deepened during the treatment, reaching $70.4 \%$ of VGPR or better after the sixth induction cycle. After induction, the CR rate of $33.4 \%$ was similar in the 92 patients with high-risk cytogenetics (34.8\%), also deepened after ASCT and consolidation $(44.1 \%$ and $50.2 \%$, respectively). Rates of minimal residual disease (MRD) also increased from induction (28.8\%) to transplantation (42.1\%) and to consolidation (45.2\%). [4]

Combinations of four drugs, including anti-CD38 monoclonal antibodies, can further improve results. The CASSIOPEIA phase 3 study was conducted in patients eligible for ASCT with newly diagnosed MM. Patients were randomized to receive four cycles of 
pre-transplantation induction and two cycles of post-transplantation consolidation of VTd alone or in combination with daratumumab (Dara-VTd). The Dara-VTD arm increased PFS and MRD response rates compared to VTD alone ( $34.6 \%$ in the VTD-daratumumab vs. arm. $23.1 \%$ in the VTD arm (p, .0001). [5]

\section{WHEN IS THE BEST TIME TO PERFORM THE TRANSPLANT?}

To evaluate the benefits of ASCT in first line compared to new drugs combinations, the EMN02 / HO95 MM Trial compare four cycles of bortezomib-melphalan-prednisone (VMP) versus melphalan $200 \mathrm{mg} / \mathrm{m} 2$ (HDM) followed by a single or double ASCT. In intention to treat analysis, the median PFS was 41.9 months in the VMP arm and 56.7 months in the HDM $\operatorname{arm}(\mathrm{HR}=0.73,0.62-0.85 ; \mathrm{p}=0.0001)$. [6]

Another study, conducted by the IFM, evaluated lenalidomide, bortezomib, and dexamethasone (RVD) in 700 patients up to 65 years old who were randomized to receive induction therapy with three cycles of RVD and then consolidation therapy with five additional cycles (350 patients) or high doses of melphalan and ASCT followed by two other cycles of RVD (350 patients). ASCT arm patients obtain a higher CR rate, PFS (50 months versus 36 months) and MRD negative rate compared to the group without transplant [7].

\section{HIGH DOSE CHEMOTHERAPY REGIMENS}

The vast majority of high-dose chemotherapy regimens used in $\mathrm{MM}$ are based on high doses of melphalan (140 to $200 \mathrm{mg} / \mathrm{m} 2$ ). Combinations, including other alkylating agents, have already been used, but none has shown significant advantages than melphalan. The use of oral busulfan and melphalan (BU-MEL) when compared to MEL200, did not offer benefits for $O S$ (77 versus 70 months, $P=0.4$ ) [8]. The association of venous busulfan $(9.6 \mathrm{mg} / \mathrm{kg})$ and melphalan (140mg / m2) is still a subject of studies [4] and can be used for high-risk patients (9).

\section{POST-TRANSPLANT STRATEGIES}

Although high doses of melphalan deepen response rates, most patients inevitably relapse. Post-transplant consolidation and maintenance are two strategies that have been used to improve responses and increase the duration of remission; however, there is still much controversy regarding the best strategies.

\subsection{CONSOLIDATION / DOUBLE TRANSPLANTATION}

The StaMINA Study was designed to assess the role of double ASCT and consolidation post-ASCT. Patients eligible for ASCT were included within 12 months after starting treatment and were randomly assigned to ASCT plus consolidation (arm 1) or double ASCT (arm 2) or a single ASCT (arm 3). All arms included maintenance with lenalidomide until progression. The results demonstrated comparable PFS and OS, suggesting that consolidation with RVD or a second ASCT was not superior to a single ASCT, followed by maintenance with lenalidomide in MM's initial treatment. [10]

The EMN02 / HO95 Study evaluated 1499 MM patients aged $\leq 65$ years eligible for ASCT. Of these, 1121 patients underwent VCD induction and then a first randomization (R1) that compared four cycles of VMP (505) versus high doses of melphalan (HDM) and single or double ASCT ( $n=706) .877$ patients underwent a second randomization (R2) for consolidation therapy with two cycles of VRD $(n=449)$ versus non-consolidation $(n=428)$, and all patients received maintenance with lenalidomide at a dose of $10 \mathrm{mg}$ continuously until progression or toxicity in both arms. The primary endpoints were PFS after R1 and R2. The PFS of R1 was favorable to ASCT vs VMP with a median of 56.7 vs 41.9 months respectively, $(\mathrm{HR}=0.73 ; \mathrm{p}=0.0001)$. PFS after R2 with adjustment for R1 was significantly prolonged in patients randomized to VRD $(\mathrm{HR}=0.77 ; 95 \% \mathrm{Cl}=0.63-0.95$; $P=0.014)$. The benefit of consolidation was seen in patients with low-risk cytogenetics $(\mathrm{HR}=0.68 ; \mathrm{P}=$ $0.03)$, but not in patients with high-risk cytogenetics (del $(17 p)$ and / or t $(4 ; 14)$ and / or t $(14 ; 16)$; HR $=1.03 ; \mathrm{P}=0.91)$. Another data emerged from this study, was the advantage in PFS and OS in favor of performing a double ASCT [6].

\subsection{MAINTENANCE}

The IFM 2005-02 study compared lenalidomide versus placebo after ASCT, with 307 patients in each arm. This trial demonstrated a clear advantage in PFS for the lenalidomide group ( $p<0.001$ ). However, there was no difference regarding OS. The CALGB study, on the other hand, demonstrated an advantage of lenalidomide maintenance in both PFS ( $p$ $<0.001)$ and $O S(p=0.03)$. [11.12].

A meta-analysis confirmed a significant improvement in PFS and OS of lenalidomide mainte- 
nance versus placebo or observation. PFS was 52.8 months for the lenalidomide group and 23.5 months for the placebo or observation group. The median OS was not achieved in the lenalidomide maintenance group and was 86 months in the placebo or observation group ( $\mathrm{HR}, 0.75 ; 95 \% \mathrm{Cl}, 0.63$ to $0.90 ; P=0.001$ ), representing a reduction of $25 \%$ in the risk of death with maintenance, benefiting all subgroups, except patients with high cytogenetic risk and ISS stage III. [13]

\section{ALLOGENIC HEMATOPOIETIC STEM CELL TRANSPLANTATION}

The role of allogeneic bone marrow transplantation (BMT) in MM's treatment remains controversial, mainly due to a high transplant-related mortality (TRM). [14] Non-myeloablative (NMA) and reduced intensity (RIC) conditioning brought the prospect of lowering TRM. Although the TRM rate reduction was achieved, there was no increase in the overall survival for patients submitted to the allogeneic BMT RIC due to the increased frequency of relapse. [14]

Several studies have evaluated the strategy of combining an autologous BMT followed by a reduced intensity allogeneic BMT (Tandem auto / RIC alo). However, a meta-analysis demonstrated that despite the high CR rates in the tandem auto / RIC alo, there was no increase in OS compared to the auto-auto tandem BMT. It occurs mainly due to a high mortality rate not related to relapse, primarily attributed to acute and chronic graft versus host disease. [15]

\section{RECOMMENDATIONS:}

- First-line treatment for patients under the age of 75 , with good PS and preserved organic functions. (Grade A of recommendation; level Ib of evidence)

- Recommended conditioning: Melphalan 200mg / $\mathrm{m} 2$ (Grade A of recommendation; level lb of evidence)

- Best moment of ASCT: upfront, after 4 to 6 cycles of induction with a combination of 3/4 drugs, including new agents, bortezomib, thalidomide, lenalidomide, daratumumab - VTD, VRD, Dara-VTD (Grade A of recommendation; level lb of evidence)

- Double transplantation as an initial strategy: Not recommended (Grade A recommendation; level la of evidence). Consider for patients with high-risk cytogenetic.

- Mobilization of PBSC: Patients responding to induc- tion treatment should be mobilized with GCSF alone. Collect a minimum cell dose of $3 \times 106$ CD34 cells / $\mathrm{kg}$. It is desirable to store cells for an eventual second ASCT; in this case, collect at least $6 \times 106$ CD34 cells $/ \mathrm{kg}$. Plerixafor is recommended for patients with GCSF mobilization failure (Grade $\mathrm{C}$ of recommendation; level IV of evidence)

- Patients with renal failure: ASCT can be recommended, with a reduced dose in conditioning. Use melphalan 100 to $140 \mathrm{mg} / \mathrm{m} 2$ (Grade C of recommendation; level IV of evidence).

- Consolidation strategies after ASCT: Two to four consolidation cycles, repeating the initial treatment (VTD or VRD or Dara-VTD), particularly for patients with no complete response after ASCT. (Grade B of recommendation; level llb of evidence)

- Maintenance strategies after ASCT: Lenalidomide until progression. (Grade A of recommendation; Ib level of evidence).

- Myeloablative allogeneic BMT or RIC can be considered for younger patients with good PS and adequate organic function who present high-risk MM (primarily refractory or less than a year of response after ASCT or with deletion of chromosome 17p). The procedure should preferably perform at a Center of Excellence. (Grade B of recommendation; level Ilb of evidence).

- RIC after auto-ASCT did not show favorable results in most clinical studies and is not recommended (Grade A of recommendation; level lb of evidence).

Clinical significance of the measurable residual disease (MRD) in multiple myeloma patients

Minimal / measurable residual disease (MRD) assessment has been considered the most important independent prognostic factor in multiple myeloma $(\mathrm{MM})$, used to assess drug efficacy and in selecting further therapeutic options in MM[16-20]. Depth response based MRD emerged as a criterion for better results in MM [21-23]. Patients who remain with detectable MRD after front-line therapy have inferior outcomes $[17,24-30]$ whereas those who achieve undetectable MRD in bone marrow (BM) have significantly improve survival [31-33]. In the context of autologous stem cell transplantation (ASCT), MRD status also provides a powerful prognostic information in MM [26-27,34], including stratification of risk relapse after HDT/ASCT (day +100)[16].

However, MM often recurs due to residual MM cells, drug resistance and/or persistence of resistant dor- 
mant subclones $[32,35]$. Therefore, more sensitive and standardized methods, such as next-generation sequencing (NGS) and next-generation flow cytometry (NGF) $[16,33,36-37]$ are needed to fulfil the MRD criteria response accordingly with the International Myeloma Working Group (IMWG) $[33,38]$ (Figure 1). Moreover, MM patients present high frequency of extramedullary relapses, not detected by BM-MRD assessment. Thus, sensitive imaging techniques such as PET-CT have become relevant in assessing low levels of disease outside BM [36-37]. Therefore, both BM-MRD and imaging techniques must be complementary to assess the response to MM treatment. In conclusion, considering the patchy pattern of BM infiltration observed in MM that leads to a degree of ambiguity regarding MRD negative results, it would be safer to make clinical decisions based on MRD positivity rather than on MRD negativity [37].

Time-points of BM-MRD assessment: MRD kinetics are more informative than single time point assessments and may be useful to address specific clinical decisions, such as early versus delayed ASCT for complete response (CR) patients after induction $[33,37]$. It allows the identification of chemosensitive (MRD-negative cases at 2 time points), intermediate, and chemoresistant patients (MRD-positive patients at 2 time points) [37].

Recommendations (not consensual): 1) at time of most optimal response (e.g. immunofixation-negative $(\mathrm{R})$; 2) before ASCT ; 3) at D+100 post-ACST; 4) after post-transplant consolidation therapy; 5 ) before the start of maintenance therapy and in subsequent time points (e.g. every 6 months), to assess the maintenance of MRD negativity achieved $[16,20,33]$.

Methods for BM-MRD assessment: flow cytometry methods do not require patient-specific diagnostic phenotypic profiles [37] as a reference, but molecular methods are based on the patient's initial specific sequences of $\mathrm{IgH}-\mathrm{VJ} / \mathrm{DJ}$ and IgK DNA regions [39]. NGF or NGS have similar sensitivity (10-5 to 10-6 neoplastic cells), high applicability, specificity and reproducibility, but their performance depends on strict rigor in the execution of the methodology [20]. NGS is a labor intensive and expensive technology, and it is yet not commonly available for clinical practice [16]

Recommendations for ensuring high quality samples for MRD detection: 1) first pull of BM aspirates [19], 2) maximum volume of $2-5 \mathrm{~mL}$ to avoid hemodilution[15,25-26]. In clinical practice, BM hemodilution needs to be recognized and reported, due to its impact on the distribution of cell populations including $\mathrm{CPC}$.
Note: the true prognostic value of the detection of MM cells in the circulation of MM patients who achieve a CR should be confirmed in prospective studies [33].

Method for disease monitoring in serum: Mass spectrometry is able to identify the $\mathrm{M}$ protein molecular mass with high precision and accuracy, allowing single clone tracking with very high sensitivity, slightly higher than NGF. It is a promising method for measuring disease activity, but it needs prospective studies to validate its applicability in clinical trials of MM $[42,43]$.

MRD assessment reports: to allow a correct interpretation of the MRD results, the MRD report must provide clear information about the MRD result and the MRD technique used, including the limits of detection and quantification achieved by the specific assay used, which are parameters of the sensitivity of the method $[44,45]$.

Status of MRD in MM and clinical practice currently: The association of MRD negativity and outcome improvement has been evidenced in both the newly diagnosed and relapsed/refractory MM patients and thus is currently considered a prognostic biomarker. However, at this time, MRD has been established as a surrogate endpoint only in clinical trials. A surrogate endpoint does not directly measure the clinical benefit of primary interest but rather is expected to predict the clinical benefit or harm based on epidemiologic, therapeutic, pathophysiologic, or other scientific evidence. Therefore, the use of MRD to make treatment decisions outside of the context of a clinical trial is not recommended [46].

\section{LIGHT CHAIN (AL) AMYLOIDOSIS}

High dose melphalan followed by rescue with autologous hematopoietic stem cells (ASCT) was introduced as a promising treatment option for light chain (AL) amyloidosis patients, but with a high mortality rate. To reduce mortality, the Mayo Clinic Group suggest a risk stratification for ASCT that is widely used and includes the following criteria: Age $\leq 70$ years, Troponin $\mathrm{t}<0.06 \mathrm{ng} / \mathrm{dl}$, NT pro-BNP $<5000 \mathrm{ng} / \mathrm{L}$, Creatinine Clearance $\geq 30 \mathrm{~mL} / \mathrm{min}$, Performance Status (ECOG) $\leq 2$, Functional Cardiac Status (New York Heart Association) classes I or II, maximum of two organic impairments (liver, kidney, heart or autonomic neurological) , absence of significant pleural effusion and lack of oxygen support. Only patients who meet all these criteria are considered potentially eligible for ASCT. [47] 
Although any recommendation in $\mathrm{AL}$ amyloidosis is controversial by the rarity and heterogeneity of the disease [48], for which randomized studies are lacking, the Andromeda Phase 3 study, for patients with no intention of transplantation, points out the Dara-CyBorD as a potential therapeutic regimen of choice for this group of patients. In this Study, 388 patients were randomized to receive CYBorD (cyclophosphamide, bortezomib and dexamethasone) or Daratumumab- CyBorD. The CR haematological rate, Major Organ Deterioration - PFS and the organ response was in favor to Dara-CyBorD. The safety profile was consistent with that previously observed for Dara SC and CyBorD. [49]

\section{GROUP RECOMMENDATIONS:}

- ASCT is the first-line treatment for patients with low-risk light chain (AL) amyloidosis. Use risk stratification criteria for this purpose (Grade B of recommendation; level Ila of evidence)

\section{REFERENCES MULTIPLE MYELOMA}

1.Hungria VT, Maiolino A, Martinez G, Duarte GO, Bittencourt R, Peters L, Pasquini R. Observational study of multiple myeloma in Latin America. Ann hematol; v. 96, n. 1, p. 65-72, 2017

2.Sonneveld P, Goldschmidt H, Rosiñol L, Blade'J, Lahuerta JJ, Cavo M, et al. Bortezomib-Based Versus Nonbortezomib-Based Induction Treatment Before Autologous Stem-Cell Transplantation in Patients With Previously Untreated Multiple Myeloma: A Meta-Analysis of Phase III Randomized, Controlled Trials. J Clin Oncol; v. 31, p. 3279-3287, 2013.

3.Moreau P, H Cyrille, Macro M, Caillot D, Chaleteix $C$, Roussel $M$, et al. VTD is superior to VCD prior to intensive therapy in multiple myeloma: results of the prospective IFM2013-04 trial. Blood; v. 127, n. 21, p. 2569-2574, 2016.

4. Rosiñol L, Oriol A, Rios R, Sureda A, Blanchard MJ, Hernandez MT, et al. Bortezomib, lenalidomide, and dexamethasone as induction therapy prior to autologous transplant in multiple myeloma. Blood. v.134, n.16, p. 1337-1345, 2019.

5.Moreau P, Attal M, Hulin C, et al. Bortezomib, thalidomide, and dexamethasone with or without daratumumab before and after autologous stem-cell transplantation for newly diagnosed multiple myeloma (CASSIOPEIA): a randomised, open-label, phase 3 study. Lancet; v. 394, n. 10192, p. 29-38, 2019
6.Cavo M, Gay F, Beksac M et al. Autologous Haematopoietic Stem-Cell Transplantation Versus Bortezomib-MelphalanPrednisone, With or Without BortezomibLenalidomide-Dexamethasone Consolidation Therapy, and Lenalidomide Maintenance for Newly Diagnosed Multiple Myeloma (EMN02/HO95): A Multicentre, Randomised, Open-Label, Phase 3 Study. Lancet Haematol 2020; 30: S2352-3026(20)30099-5. doi: 10.1016/ S2352-3026(20)30099-5. [Epub ahead of print].

7.Attal M, Lauwers-Cances V, Hulin C, Leleu X, Caillot $D$, Escoffre $M$, et al. Lenalidomide, Bortezomib, and Dexamethasone with Transplantation for Myeloma. N Engl J Med; v. 376, p.1311-1320, 2017.

8.Lahuerta JJ, Mateos MV, Martínez-López J, et al. Busulfan $12 \mathrm{mg} / \mathrm{kg}$ plus melphalan $140 \mathrm{mg} /$ $\mathrm{m} 2$ versus melphalan $200 \mathrm{mg} / \mathrm{m} 2$ as conditioning regimens for autologous transplantation in newly diagnosed multiple myeloma patients included in the PETHEMA/GEM2000 study. Haematologica; v. 95, n. 11, p. 1913-1920, 2010.

9.Saini N, Bashir Q, Milton DR, et al. Busulfan and melphalan alone in autologous stem cell transplantation for high-risk MM. Blood Adv; v. 4, n. 19, p. 4834-4837, 2020.

10.Stadtmauer EA, Pasquini MC, Blackwell B, et al. Autologous Transplantation, Consolidation, and Maintenance Therapy in Multiple Myeloma: Results of the BMT CTN 0702 Trial. J Clin Oncol; v. 37, n.7, p. 589-597, 2019.

11.Attal M, Lauwers-Cances V, Marit G, Caillot D, Moreau P, Facon T, et al, IFM Investigators. Lenalidomide maintenance alter stem-cell transplantation for multiple myeloma. N Engl J Med; 2012 v.366, n.19, p. 1782- 1791, 10 may 2012.

12.McCarthy PL, Owzar K, Hofmeister CC, Hurd $D D$, Hassoun $\mathrm{H}$, Richardson PG, et al. Lenalidomide alter stem-cell transplantation for multiple myeloma. N Engl J Med; v. 366, n. 19, p. 1770-1781, 2012.

13.McCarthy PL, Holstein SA, Petrucci MT et al. Lenalidomide Maintenance After Autologous Stem-Cell Transplantation in Newly Diagnosed Multiple Myeloma: A Meta-Analysis. J Clin Oncol; v. 35, p. 3279-3289, 2017.

14.Dhakal B, Vesole DH, Hari PN. Allogeneic stem cell transplantation for multiple myeloma:ls there a future? Bone Marrow Transplant; v. 51, n. 4, p. 2016. 
15.Shah N, Callander N, Ganguly S, et al. Hematopoietic Stem Cell Transplantation for multiple Myeloma: Guidelines from the American Society for Blood and Marrow Transplantation. Biol Blood Transplant; v. 21, p. 2015.

\section{REFERENCES MINIMAL RESIDUAL DISEASE}

16.Paiva B, Puig N, García-Sanz R, San Miguel JF. Is this the time to introduce minimal residual disease in multiple myeloma clinical practice? Clinical Cancer Research; v. 21, n. 9, p. 2015.

17.Lahuerta JJ, Paiva B, Vidriales MB, Cordón L, Cedena MT, Puig N, et al. Depth of response in multiple myeloma: A pooled analysis of three PETHEMA/GEM clinical trials. Journal of Clinical Oncology; it. v. 35, n.25, p. 2017.

18.Munshi NC, Avet-Loiseau H, Rawstron AC, Owen RG, A CJ. Minimal residual disease predicts superior survival in patients with multiple myeloma: a meta-analysis. JAMA Oncology. v. 3, n.1, p. 2835, 2017.

19.Roshal M. Minimal Residual Disease Detection by Flow Cytometry in Multiple Myeloma: Why and How? Seminars in Hematology [Internet]; v. 55, n. 1, p.4-12, 2018. Available from: https://doi. org/10.1053/j.seminhematol.2018.02.011.

20.Romano A, Palumbo GA, Parrinello NL, Conticello $C$, Martello M, Terragna C. Minimal Residual Disease Assessment Within the Bone Marrow of Multiple Myeloma: A Review of Caveats, Clinical Significance and Future Perspectives. Frontiers in Oncology; v. 9, p.1-14, 2019.

21.Lahuerta JJ, Paiva B, Vidriales MB, Cordón L, Cedena MT, Puig N, et al. Depth of response in multiple myeloma: A pooled analysis of three PETHEMA/GEM clinical trials. Journal of Clinical Oncology; v. 35, n. 25, p. 2900-10, 2017.

22.Munshi NC, Anderson KC, Bergsagel PL, Shaughnessy J, Palumbo A, Durie B, et al. Consensus recommendations for risk stratification in multiple myeloma : report of the International Myeloma Workshop Consensus Panel 2 Consensus recommendations for risk stratification in multiple myeloma : report of the International Myeloma Workshop Con; v. 117, n. 18, p. 4696-700, 2012.

23. Harousseau JL, Avet-Loiseau H. Minimal residual disease negativity is a new end point of myeloma therapy. Journal of Clinical Oncology; v. 35, n. 25, p. 2017.
24.Scott SD, Fletcher $M$, Whitehouse $H$, Whitby $L$, Yuan C, Mazzucchelli S, et al. Assessment of plasma cell myeloma minimal residual disease testing by flow cytometry in an international inter-laboratory study: Is it ready for primetime use? Cytometry Part B - Clinical Cytometry; v. 96, n. 3, p. 2019.

25.Paiva B, Cedena MT, Puig N, Arana P, Vidriales $M B$, Cordon $L$, et al. Minimal residual disease monitoring and immune profiling in multiple myeloma in elderly patients; Blood; v. 127, $\mathrm{n}$. 25,p.2016.

26.Paiva B, Vidriales MB, Cerveró J, Mateo G, Pérez $J$ J, Montalbán MA, et al. Multiparameter flow cytometric remission is the most relevant prognostic factor for multiple myeloma patients who undergo autologous stem cell transplantation. Blood. v. 112,n.10, p. 4017- 4023, 2008.

27.De Tute RM, Rawstron AC, Gregory WM, Child JA, Davies FE, Bell SE, et al. Minimal residual disease following autologous stem cell transplant in myeloma: Impact on outcome is independent of induction regimen. Haematologica. v. 101, n.2, p. 69-71, 2016.

28.Martínez-Sánchez P, Montejano L, Sarasquete ME, García-Sanz R, Fernández-Redondo E, Ayala $R$, et al. Evaluation of minimal residual disease in multiple myeloma patients by fluorescent-polymerase chain reaction: The prognostic impact of achieving molecular response. British Journal of Haematology; v. 142, n. 5, p.766-774, 2008.

29.Landgren O, Devlin S, Boulad M, Mailankody S. Role of MRD status in relation to clinical outcomes in newly diagnosed multiple myeloma patients: A meta-analysis. Bone Marrow Transplantation; v.51, n. 12, 2016.

30.Flores-Montero J, Sanoja-Flores L, Paiva B, Puig N, García-Sánchez O, Böttcher S, et al. Next Generation Flow for highly sensitive and standardized detection of minimal residual disease in multiple myeloma. Leukemia; v. 31, n. 10, p. 2094- 403, 2017.

31. Mateos M-V, Ocio EM, Paiva B, Rosiñol L, Martínez-López J, Bladé J, et al. Treatment for patients with newly diagnosed multiple myeloma in 2015. Blood Reviews [Internet]; v. 29, n. 6, p. 387-403, 2015. Available from: http:// www.sciencedirect.com/science/article/pii/ S0268960X15000430

32.Rovira M, Blade J. Pattern of relapse and progres- 
sion after autologous SCT as upfront treatment for multiple myeloma; p. 223-227, july 2014.

33.Kumar S, Paiva B, Anderson KC, Durie B, Landgren $\mathrm{O}$, Moreau $\mathrm{P}$, et al. International Myeloma Working Group consensus criteria for response and minimal residual disease assessment in multiple myeloma. Lancet Oncology [Internet]. v. 17, n. 8, p. 328-346. Available from: http://dx.doi. org/10.1016/S1470-2045(16)30206-6

34.Paiva B, van Dongen JJ, Orfao A. New criteria for response assessment: role of minimal residual disease in multiple myeloma. Blood; v. 125, n. 20, p. 3059-3068, 2015.

35.Paiva B, Corchete LA, Vidriales M-B, Puig N, Maiso $\mathrm{P}$, Rodriguez I, et al. Phenotypic and genomic analysis of multiple myeloma minimal residual disease tumor cells: a new model to understand chemoresistance. Blood; v. 127, n.15, p. 18961906, 2016.

36.Caers J, Garderet L, Kortüm KM, O'Dwyer ME, van de Donk NWCJ, Binder M, et al. European Myeloma Network recommendations on tools for the diagnosis and monitoring of multiple myeloma: what to use and when. Haematologica; v.103, n.11, p.1772-1784, 2018.

37.Fulciniti M, Munshi NC, Martinez-Lopez J. Deep Response in Multiple Myeloma: A Critical Review. BioMed Research International 2015, http:// dx.doi.org/10.1155/2015/832049

38.Martínez-López J, Paiva B, López-Anglada L, Mateos MV, Cedena T, Vidríales MB, et al. Critical analysis of the stringent complete response in multiple myeloma: Contribution of sFLC and bone marrow clonality. Blood; v. 126, n. 7, p. 858-862, 2015.

39.Takamatsu H. Comparison of Minimal Residual Disease Detection by Multiparameter Flow Cytometry, ASO-qPCR, Droplet Digital PCR, and Deep Sequencing in Patients with Multiple Myeloma Who Underwent Autologous Stem Cell Transplantation. J. Clin. Med; v. 6, n. 91, p. 1-11, 2017.

40.Ciudad J, Orfao A, Vidriales B, Macedo A, Martínez A, González M, et al. Immunophenotypic analysis of CD19+ precursors in normal human adult bone marrow: Implications for minimal residual disease detection. Haematologica; v. 83, n. 12, p. 1069-75, 1998.
41.Delgado JA, Guillén-Grima F, Moreno C, Panizo C, Pérez-Robles C, Mata JJ, et al. A simple flow-cytometry method to evaluate peripheral blood contamination of bone marrow aspirates. Journal of Immunological Methods [Internet]; v. 442, p. 54-58, 2017. Available from: http://dx.doi. org/10.1016/j.jim.2016.12.006

42.Thoren KL. Mass spectrometry methods for detecting monoclonal immunoglobulins in multiple myeloma minimal residual disease. Seminars in Hematology [Internet]; v. 55, n. 1, p. 41-3, 2018. Available from: https://doi.org/10.1053/j. seminhematol.2018.02.008

43.Puig N, Mateos MV, Contreras T, Paiva B, Cedena MT, Pérez JJ et al. Qip-Mass Spectrometry in High Risk Smoldering Multiple Myeloma Patients Included in the GEM-CESAR Trial: Comparison with Conventional and Minimal Residual Disease IMWG Response Assessment. Blood. p. 134, 2019. https://doi.org/10.1182/ blood-2019-127717

44.Arroz M, Came N, Lin P, Chen W, Yuan C, Lagoo A, et al. Consensus Guidelines on Plasma Cell Myeloma Minimal Residual Disease Analysis and Reporting. Cytometry Part B; p. 31-39, 2016.

45.Clinical and Laboratory Standards Institute (CLSI). Evaluation of Detection Capability for Clinical Laboratory Measurement Procedures; Approved Guideline, 2nd ed. CLSI document EP17-A2 (ISBN 1-56238-795-2). Pennsylvania: Clinical and Laboratory Standards Institute; 2012.

46. Holstein AS, Al-Kadhimi Z, Costa LJ, Hahn T, Hari $P$, Hillengass J, et al. Summary of the Third Annual Blood and Marrow Transplant Clinical Trials Network Myeloma Intergroup Workshop on Minimal Residual Disease and Immune Profiling. Biology of Blood and Marrow Transplantation. https://doi.org/10.1016/j.bbmt.2019.09.015

47. How to manage primary amyloidosis. Gertz M. Leukemia; v.26, p. 191-8, 2012.

48.Dispenzieri A, Mayo Clin Proc; v. 90, n. 8, p. 10541081, 2015.

49.Palladini G, Kastritis E, Maurer MS, et.al. Daratumumab plus CyBorD for patients with newly diagnosed $A L$ amyloidosis:safety run-in results of ANDROMEDA. Blood; v. 136, n. 1, p. 71-80, 2020. 